Pacific Journal of Mathematic 


\title{
ON SPACES WHOSE STONE-ČECH COMPACTIFICATION IS OZ
}

\author{
TOSHIJI TERADA
}

\begin{abstract}
A Tychonofi space $X$ is called $\mathrm{Oz}$ if every open subset is $z$-embedded in $X$. In this paper we characterize a class of spaces whose Stone-Čech compactifications are Oz. Especially it is shown that for a realcompact Oz-space of countable type, $\beta X$ is $0 z$ if and only if $X$ is expressed as the union of an extremally disconnected subset and a compact subset.
\end{abstract}

1. Introduction. All spaces considered here are Tychonoff. A subset $S$ of a space $X$ is $z$-embedded in $X$ in case each zero-set of $S$ is the restriction to $S$ of a zero-set of $X$. A space $X$ is called an Oz-space if every open subset of $X$ is $z$-embedded in $X$. Perfectly normal spaces and extremally disconnected spaces are $\mathrm{Oz}$. For basic results of Oz-spaces, see [2] and [6]. Especially R. L. Blair [2] showed the following result: A space $X$ is an Oz-space if and only if $\nu X$ is $0 z$, where $\nu X$ is the Hewitt realcompactification of $X$. However it is unknown whether the Stone-Čech compactification $\beta X$ of an $\mathrm{Oz}$-space $X$ is $\mathrm{Oz}$.

The purpose of this paper is to characterize a class of spaces whose Stone-Čech compactifications are Oz. As an application of our characterizations it will be shown that both $\beta R$ and $\beta Q$ are not $\mathrm{Oz}$, where $R$ is the space of all real numbers and $Q$ is the space of all rational numbers. In $\S 2$, we will show formal characterizations. In $\S 3$, structural characterizations will be studied. For example, it will be shown that for a realcompact Oz-space $X$ of countable type, $\beta X$ is $\mathrm{Oz}$ if and only if $X$ can be expressed as the union of an extremally disconnected subset and a compact subset.

2. Formal characterizations. The following lemmas are basic for our studies.

Lemma 1 (R. L. Blair [2]). A space $X$ is an Oz-space if and only if every regular closed subset of $X$ is a zero-set in $X$.

Lemma 2. Let $X$ be a dense subspace of a space $Y$.

(1) If $A$ is a regular closed subset of $X$, then $\mathrm{Cl}_{Y} A$ is a regular closed subset of $Y$.

(2) If $B$ is a regular closed subset of $Y$, then $B \cap X$ is a regular closed subset of $X$. 
Lemma 2 is well-known. Let $U$ be an open subset of a space $X$. Then $\beta X-\mathrm{Cl}_{\beta X}(X-U)$ is denoted by $U^{\beta}$ in this paper.

Lemma 3 (E. G. Skljarenko [5]). For any open subset $U$ of a space $X$, the equality $\mathrm{Bd}_{\beta, Y}\left(U^{\beta}\right)=\mathrm{Cl}_{\beta X}\left(\mathrm{Bd}_{X} U\right)$ holds.

The following lemma is used only once for the proof of Theorem 1.

Lemma 4 (D. Rudd [4]). For a zero-set $Z$ of a space $X$ the following are equivalent.

(1) $\mathrm{Cl}_{\beta X} Z$ is a zero-set of $\beta X$.

(2) There exists a real-valued continuous function $f$ on $X$ with the following properties; (a) $Z=f^{-1}(0)$.

(b) If a subset $A$ of $X$ is completely separated from $Z$, then $\inf \{f(a): a \in A\}>0$.

The following theorem can be established by a routine argument relying on Lemmas 1,2 , and 4 .

THEOREM 1. For an Oz-space $X$ the following are equivalent.

(1) $\beta X$ is $O z$.

(2) For each regular closed subset $A$ of $X$ there is a sequence $\left\{U_{i}: i<\omega\right\}$ of cozero-sets of $X$ with the following properties; (a) $A \subset U_{i}$ for each $i<\omega$. (b) For any cozero-set $U$ of $X$ containing $A$ there is some $U_{i}$ such that $U_{i} \subset U$.

Another formal characterization is given as follows. This characterization is useful for the studies in $\S 3$.

THEOREM 2. For an Oz-space $X$ the following are equivalent.

(1) $\beta X$ is $O z$.

(2) For each regular closed subset $A$ of $X$ there is a sequence $\left\{U_{i}: i<\omega\right\}$ of regular open subsets of $X$ with the following properties; (a) $A \subset U_{i}$ for each $i<\omega$. (b) For any regular open subset $U$ of $X$ containing $A$ there is some $U_{i}$ such that $U_{i} \subset U$.

Proof. (1) $\rightarrow(2)$. Let $A$ be a regular closed subset of $X$. Then by Lemma $2 \mathrm{Cl}_{\beta X} A$ is a regular closed subset of $\beta X$. Hence $\mathrm{Cl}_{\beta X} A$ has a countable neighborhood basis $\left\{V_{i}: i<\omega\right\}$ consisting of regular open subsets of $\beta X$ since $\beta X$ is a compact Oz-space. For each $i<\omega$ let $U_{i}=V_{i} \cap X$. Then it will be shown that $\left\{U_{i}: i<\omega\right\}$ has the properties (a) and (b). (a) is obviously satisfied. Let $U$ be a regular open subset of $X$ containing $A$. Then $A$ and $X-U$ are 
completely separated since $A$ and $X-U$ are regular closed subsets of an Oz-space $X$. Hence $\mathrm{Cl}_{\beta X} A \subset U^{\beta}$. Therefore, for some $i, \mathrm{Cl}_{\beta_{X}} A \subset$ $V_{i} \subset U^{\beta}$. Thus $U_{i} \subset U$ for some $i$. Hence (b) is satisfied.

$(2) \rightarrow(1)$. Let $B$ be a regular closed subset of $\beta X$. Then $A=$ $B \cap X$ is a regular closed subset of $X$. Hence there is a sequence $\left\{U_{i}: i<\omega\right\}$ of regular open subsets of $X$ with the properties (a) and (b). Then it is obvious that $\mathrm{Cl}_{\beta X} A=B=\cap\left\{U_{i}^{\beta}: i<\omega\right\}$. Hence $B$ is a zero-set of $\beta X$ since $\beta X$ is normal. This completes the proof.

CoRollary 1. For a normal space $X$ the following are equivalent.

(1) $\beta X$ is $O z$.

(2) Every regular closed subset of $X$ has a countable neighborhood basis.

CoROllary 2. $\beta R, \beta Q$ and $\beta(R-Q)$ are not $O z$.

3. Structural characterizations. A subset $S$ of a space $X$ is called relatively pseudocompact if every real-valued continuous function $f$ on $X$ satisfies the condition that the restriction $f \mid S$ is bounded.

THEOREM 3. If $\beta X$ is $O z$, then for any regular closed subset $A$ of $X, \mathrm{Bd}_{X} A$ is relatively pseudocompact.

Proof. Let $A$ be a regular closed subset of $X$. Assume that $\mathrm{Bd}_{X} A$ is not relatively pseudocompact. Then it will be proved that condition (2) of Theorem 2 is not satisfied. Let $\left\{U_{i}: i<\omega\right\}$ be a sequence of regular open subsets of $X$ containing $A$. Since $\operatorname{Bd}_{X} A$ is not relatively pseudocompact, $\mathrm{Cl}_{\beta X}\left(\mathrm{Bd}_{X} A\right) \cap(\beta X-v X)$ is nonempty. Let $y$ be a point of $\mathrm{Cl}_{\beta X}\left(\mathrm{Bd}_{X} A\right) \cap(\beta X-v X)$. Then it is obvious that $y \in \mathrm{Cl}_{\beta X}\left(U_{i}-A\right)$ for each $i<\omega$. Since $y \notin \nu X$, there is a discrete sequence $\left\{F_{i}: i<\omega\right\}$ of regular closed subsets of $X$ such that $F_{i} \subset U_{i}-A$ for each $i<\omega$. Now let $U=X-\cup\left\{F_{i}: i<\omega\right\}$. Then $U$ is a regular open subsets of $X$ containing $A$. But $U$ contains no member of $\left\{U_{i}: i<\omega\right\}$ by the construction.

CoRollary 3. If $\beta X$ is $O z$, then the following hold.

(1) $\operatorname{ind}(\beta X-\nu X) \leqq 0$.

(2) For any regular open subset $U$ of $\nu X, \mathrm{Bd}_{\nu X} U$ is compact.

A space $X$ is called of countable type if, for any compact subset $C$ of $X$, there is a compact subset $C^{\prime}$ such that $C \subset C^{\prime}$ and $C^{\prime}$ has a countable neighborhood basis (see [1]). 
THEOREM 4. If $\cup X$ is of countable type, then the following are equivalent.

(1) $\beta X$ is $O z$.

(2) For any regular closed subset $A$ of $X, \mathrm{Bd}_{X} A$ is a relatively pseudocompact zero-set.

Proof. (1) $\rightarrow(2)$. Since $X$ must be $\mathrm{Oz}, \mathrm{Bd}_{X} A$ is a zero-set for any regular closed subset $A$ of $X$. Then by Theorem 3 this implication is obvious.

$(2) \rightarrow(1)$. Let $B$ be a regular closed subset of $\beta X$. Then $B \cap X$ is a regular closed subset of $X$. Hence $\operatorname{Bd}_{X}(B \cap X)$ is a relatively pseudocompact zero-set of $X$. Since $\mathrm{Bd}_{\beta X} B=\mathrm{Cl}_{\beta X}\left(\mathrm{Bd}_{x}(B \cap X)\right), \mathrm{Bd}_{\beta X} B$ is a compact zero-set of $v X$. By the assumption that $\nu X$ is of countable type, $\mathrm{Bd}_{\beta X} B$ is a $G_{\delta}$-set of $\beta X$. Thus $B$ is $G_{\delta}$ in $\beta X$.

Next, we will show that, in Corollary 1, the normality of $X$ can be replaced by the realcompactness of $X$.

Lemma 5. Let $X$ be a realcompact space and let $A$ be a closed subset of $X$. If $A$ has a countable neighborhood basis in $X$, then $\mathrm{Cl}_{\beta X} A$ is a zero-set of $\beta X$.

Proof. Let $\left\{U_{i}: i<\omega\right\}$ be a countable neighborhood basis of $A$. Assume that $\mathrm{Cl}_{\beta X} A-U_{i_{0}}^{\beta} \neq \varnothing$ for some $i_{0}$. Then since $\mathrm{Cl}_{\beta X} A \subset$ $\left(U_{i_{0}} \cap U_{i}\right)^{\beta} \cup \mathrm{Bd}_{\beta X}\left(\left(U_{i_{0}} \cap U_{i}\right)^{\beta}\right)=\left(U_{i_{0}} \cap U_{i}\right)^{\beta} \cup \mathrm{Cl}_{\beta X}\left(\mathrm{Bd}_{X}\left(U_{i_{0}} \cap U_{i}\right)\right) \subset U_{i_{0}}^{\beta} \cup$ $\mathrm{Cl}_{\beta X}\left(U_{i}-A\right)$ for each $i<\omega, \mathrm{Cl}_{\beta X} A-U_{i_{0}}^{\beta} \subset \mathrm{Cl}_{\beta X}\left(U_{i}-A\right)$ for each $i<\omega$. If we take a point $y$ in $\mathrm{Cl}_{\beta X} A-U_{i_{0}}^{\beta}$, then by the same argument in the proof of Theorem 3 it is shown that $\left\{U_{i}: i<\omega\right\}$ is not a neighborhood basis of $A$ in $X$. This is a contradiction. Thus $\mathrm{Cl}_{\beta X} A \subset U_{i}^{\beta}$ for each $i<\omega$. Then it is obvious that $\mathrm{Cl}_{\beta X} A=\cap$ $\left\{U_{i}^{\beta}: i<\omega\right\}$. Thus $\mathrm{Cl}_{\beta X} A$ is a zero-set of $\beta X$.

CoRollary 4. Let $X$ be a realcompact space. If every closed subset of $X$ has a countable neighborhood basis, then $X$ is (perfectly) normal.

THEOREM 5. For a realcompact space $X$ the following are equivalent.

(1) $\beta X$ is $O z$.

(2) Any regular closed subset $A$ of $X$ has a countable neighborhood basis in $X$.

(3) For any regular closed subset $A$ of $X, \mathrm{Bd}_{X} A$ is a compact subset with a countable neighborhood basis in $X$. 
Proof. (1) $\rightarrow(3)$. By Lemma 3, for any regular closed subset $A$ of $X, \mathrm{Cl}_{\beta X} A=\mathrm{Cl}_{\beta X}\left(\mathrm{Bd}_{X} A\right) \cup\left(\operatorname{Int}_{X} A\right)^{\beta}$ and $\mathrm{Cl}_{\beta X}(X-A)=\mathrm{Cl}_{\beta_{X}}\left(\mathrm{Bd}_{X}(X-A)\right) \cup$ $(X-A)^{\beta}=\mathrm{Cl}_{\beta X}\left(\mathrm{Bd}_{X} A\right) \cup(X-A)^{\beta}$. Thus $\mathrm{Cl}_{\beta X} A \cap \mathrm{Cl}_{\beta X}(X-A)=$ $\mathrm{Cl}_{\beta X}\left(\mathrm{Bd}_{X} A\right)$. Therefore $\mathrm{Cl}_{\beta X}\left(\mathrm{Bd}_{X} A\right)$ is $G_{\tilde{o}}$ in $\beta X$ since $\beta X$ is Oz. By Theorem $3, \mathrm{Bd}_{X} A$ is relatively pseudocompact in $X$. Since $X$ is realcompact, $\mathrm{Bd}_{X} A$ must be compact. Hence $\mathrm{Bd}_{X} A$ has a countable neighborhood basis in $X$.

$(3) \rightarrow(2)$. This is obvious.

$(2) \rightarrow(1)$. By Lemma 5 it is proved that every regular closed subset of $\beta X$ is a zero-set of $\beta X$.

A space $X$ is called extremally disconnected if the closure of every open subset is open. If $X$ is extremally disconnected or pseudocompact $\mathrm{Oz}$, then $\beta X$ is $\mathrm{Oz}$ (see [2]). Conversely we have the following.

THEOREM 6. If $\beta X$ is $O z$, then for each discrete sequence $\left\{U_{i}: i<\omega\right\}$ of open subsets of $X$ there exists $i_{0}$ such that $U_{j}$ is extremally disconnected for each $j \geqq i_{0}$.

Proof. Assume the contrary. Then there is a subsequence $\left\{U_{i_{k}}: k<\omega\right\}$ of $\left\{U_{i}: i<\omega\right\}$ such that $U_{i_{k}}$ is not extremally disconnected for each $k$. For each $k$ let $V_{k}$ be an open subset of $U_{i_{k}}$ such that $\mathrm{Cl}_{U_{i_{k}}} V_{k}$ is not open. Let $F=\cup\left\{\mathrm{Cl}_{X} V_{k}: k<\omega\right\}$. Then obviously $F$ is regular closed. But we will show that condition (2) of Theorem 2 is not satisfied. Let $\left\{W_{i}: i<\omega\right\}$ be a sequence of regular open subsets of $X$ containing $F$. Then, for each $k$, there is a regular closed subset $S_{k}$ of $X$ such that $S_{k} \subset\left(W_{k} \cap U_{i_{k}}\right)-F$. Let $U=X-\cup\left\{S_{k}: k<\omega\right\}$. Then $U$ is a regular open subset of $X$ which contains no member of $\left\{W_{i}: i<\omega\right\}$.

CoRollary 5. If every open subset of a space $X$ is not extremally disconnected, then the following are equivalent.

(1) $\beta X$ is $O z$.

(2) $X$ is pseudocompact and $O z$.

The fact that $\beta R, \beta Q$ and $\beta(R-Q)$ are not $\mathrm{Oz}$ follows also from Corollary 5. The following is the main theorem in this section.

THEOREM 7. Let $X$ be an Oz-space whose Hewitt realcompactification $\cup X$ is of countable type. Then the following are equivalent.

(1) $\beta X$ is $O z$.

(2) $X$ is expressed as the union of an extremally disconnected open subset and a relatively pseudocompact (closed) subset. 
Proof. (1) $\rightarrow(2)$. Let $\mathscr{C}$ be the family of all extremally disconnected open subsets of $X$. Then $\mathscr{C}$ is partially ordered by the inclusion relation $\subset$. Let $\mathscr{U}^{\prime}$ be a linearly ordered subset of $\mathscr{U}$. Then it is not so difficult to see that $U\left\{U: U \in \mathscr{U}^{\prime}\right\}$ is also a member of $\mathscr{U}$. Hence by Zorn's lemma there exists a maximal member $E$ of $\mathscr{Q}$. Let $P=X-E$. Assume that $P$ is not relatively pseudocompact. Then there is a discrete sequence $\left\{U_{i}: i<\omega\right\}$ of open subsets of $X$ such that $U_{i} \cap P \neq \varnothing$ for each $i$. If $U_{i}$ is extremally disconnected, then $U_{i} \cup E$ is also extremally disconnected. But this contradicts the maximality of $E$. Hence each $U_{i}$ is not extremally disconnected. But this is a contradiction by Theorem 6 . Thus $P$ is relatively pseudocompact.

$(2) \rightarrow(1)$. Let $X=E \cup P$, where $E$ is an extremally disconnected open subset and $P$ is a closed relatively pseudocompact subset. We will show that for each regular closed subset $A$ of $X, \mathrm{Bd}_{X} A$ is relatively pseudocompact. Then by Theorem 4 it is true that $\beta X$ is $\mathrm{Oz}$. It suffices to show that $\mathrm{Bd}_{x} A \subset P$. This follows from the following observation:

$$
\begin{aligned}
\operatorname{Bd}_{X} A= & \mathrm{Cl}_{X}\left(\operatorname{Int}_{X} A\right)-\operatorname{Int}_{X} A \\
= & \mathrm{Cl}_{X}\left(\left(\left(\operatorname{Int}_{X} A\right) \cap E\right) \cup\left(\left(\operatorname{Int}_{X} A\right) \cap P\right)\right)-\operatorname{Int}_{X} A \\
= & \left(\operatorname{Cl}_{X}\left(\operatorname{Cl}_{E}\left(\left(\operatorname{Int}_{X} A\right) \cap E\right)\right)-\operatorname{Int}_{X} A\right) \cup\left(\operatorname{Cl}_{X}\left(\left(\operatorname{Int}_{X} A\right) \cap P\right)\right. \\
& \left.-\operatorname{Int}_{X} A\right) \\
\subset & P \cup P \\
= & P .
\end{aligned}
$$

This completes the proof.

CoROllary 6. Let $X$ be a realcompact Oz-space of countable type. Then the following are equivalent.

(1) $\beta X$ is $O z$.

(2) $X$ is expressed as the union of an extremally disconnected subset and a compact subset.

EXAMPLE. In Theorem 4 and Theorem 7, the assumption that $v X$ is of countable type can not be omitted. In fact, there is a realcompact $\mathrm{Oz}$-space $X$ with the following properties:

(a) $X=E \cup C$, where $E$ is an extremally disconnected subset and $C$ is a compact subset.

(b) $\beta X$ is not $\mathrm{Oz}$.

Let $N$ be a countably infinite discrete space and let $p$ be a point of $\beta N-N$. Then $N \cup\{p\}$ is realcompact as a subspace of $\beta N$. Let $X$ be the quotient space of the topological sum of $N \cup\{p\}$ and the unit interval $I$ obtained by identifying the point $p$ of $N \cup\{p\}$ 
and the point 0 of $I$. Then $X$ is realcompact and $\mathrm{Oz}$ since $X$ is Lindelöf and perfectly normal. It is also obvious that $X$ can be expressed as the union of a discrete subset and a compact subset. But $\beta X$ is not $O z$ since the homeomorphic image of $I$ is a regular closed subset of $X$ which does not have a countable neighborhood basis in $X$ (see Theorem 5).

\section{REFERENCES}

1. A. V. Arhangel'skii, Bicompact sets and the topology of spaces, Trans. Moscow Math. Soc., (1965), 1-62.

2. R. L. Blair, Spaces in which special sets are z-embedded, Canad. J. Math., 28 (1976), 673-690.

3. L. Gillman and M. Jerison, Rings of continuous functions, Van Nostrand, Princeton, 1960.

4. D. Rudd, A note on zero-sets in the Stone-Čech compactification, Bull. Austral. Math. Soc., 12 (1975), 227-230.

5. E. G. Skljarenko, Some questions in the theory of bicompactifications, Izv. Akad. Nauk SSSR, 26 (1962), 427-452; Amer. Math. Soc. Transl. Ser., (2) 58 (1966), 216-244.

6. T. Terada, Note on $z-, C^{*-}$, and C-embedding, Sci. Rep. Tokyo Kyoiku Daigaku Sect. A, 13 (1975), 129-132.

Received March 14, 1978 and in revised form January 8, 1979.

UNIVERSITY OF TSUKUBA

SAKURAMURA, JAPAN

Current address: Department of Mathematics

Faculty of Engineering

Yokohama National University

Yokohama, Japan 



\section{PACIFIC JOURNAL OF MATHEMATICS}

\section{EDITORS}

DONALD BABBITT (Managing Editor)

University of California

Los Angeles, California 90024

HUgo RossI

University of Utah

Salt Lake City, UT 84112

C. C. MOORE and ANDREW OGG

University of California

Berkeley, CA 94720

\section{J. DUGUNDJI}

Department of Mathematics University of Southern California Los Angeles, California 90007

R. Finn and J. Milgram Stanford University Stanford, California 94305

\section{ASSOCIATE EDITORS}

E. F. BECKENBACH

B. H. NEUMANN

F. WOLF

K. YoshidA

\section{SUPPORTING INSTITUTIONS}

UNIVERSITY OF BRITISH COLUMBIA CALIFORNIA INSTITUTE OF TECHNOLOGY UNIVERSITY OF CALIFORNIA MONTANA STATE UNIVERSITY UNIVERSITY OF NEVADA, RENO NEW MEXICO STATE UNIVERSITY OREGON STATE UNIVERSITY UNIVERSITY OF OREGON
UNIVERSITY OF SOUTHERN CALIFORNIA STANFORD UNIVERSITY UNIVERSITY OF HAWAII UNIVERSITY OF TOKYO UNIVERSITY OF UTAH WASHINGTON STATE UNIVERSITY UNIVERSITY OF WASHINGTON 


\section{Pacific Journal of Mathematics}

Vol. 85, No. $1 \quad$ September, 1979

Ralph Alexander, Metric averaging in Euclidean and Hilbert spaces...... 1

B. Aupetit, Une généralisation du théorème de Gleason-Kahane-Żelazko

pour les algèbres de Banach..............................

Lung O. Chung, Jiang Luh and Anthony N. Richoux, Derivations and

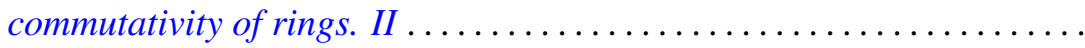

Lynn Harry Erbe, Integral comparison theorems for third order linear

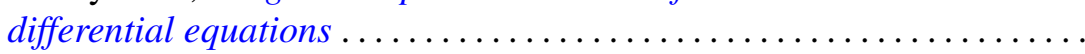

Robert William Gilmer, Jr. and Raymond Heitmann, The group of units of a

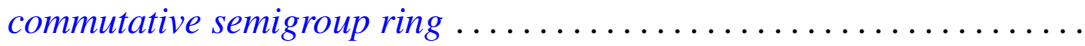

George Grätzer, Craig Robert Platt and George William Sands, Embedding lattices into lattices of ideals ...........................

Raymond D. Holmes and Anthony Charles Thompson, $n$-dimensional area and content in Minkowski spaces ....................... 77

Harvey Bayard Keynes and M. Sears, Modelling expansion in real flows....

Taw Pin Lim, Some classes of rings with involution satisfying the standard

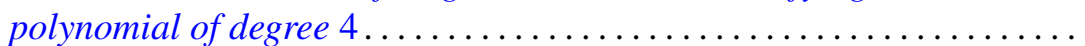

Garr S. Lystad and Albert Robert Stralka, Semilattices having bialgebraic congruence lattices ................................... 131

Theodore Mitchell, Invariant means and analytic actions . . . . . . . . . 145

Daniel M. Oberlin, Translation-invariant operators of weak type ........ 155

Raymond Moos Redheffer and Wolfgang V. Walter, Inequalities involving

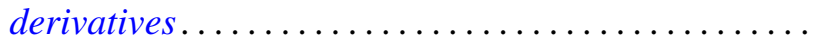

Eric Schechter, Stability conditions for nonlinear products and semigroups ................................

Jan Søreng, Symmetric shift registers ........................ 201

Toshiji Terada, On spaces whose Stone-Čech compactification is $\mathrm{Oz} \ldots \ldots .231$

Richard Vrem, Harmonic analysis on compact hypergroups ... 